\title{
Förster Resonance Energy Transfer Based Biosensor for Targeting the hNTH1-YB1 Interface as a Potential Anticancer Drug Target
}

Muge Senarisoy ${ }^{1}$, Caroline Barette ${ }^{2}$, Françoise Lacroix ${ }^{1}$, Salvatore De Bonis ${ }^{1}$, Meike Stelter ${ }^{1}$, Fabienne Hans ${ }^{1}$, Jean-Philippe Kleman ${ }^{1}$, Marie-Odile Fauvarque ${ }^{2}$ and Joanna Timmins ${ }^{1 *}$.

${ }^{1}$ Univ. Grenoble Alpes, CEA, CNRS, IBS, F-38000 Grenoble, France.

${ }^{2}$ Univ. Grenoble Alpes, CEA, INSERM, BGE, F-38000 Grenoble, France.

*Corresponding author: Joanna Timmins (Joanna.timmins@ibs.fr)

\section{Supplemental Information}

Supplemental Table S1

Supplemental Figures S1-S8 
Table S1. Potential hits identified in the Prestwick Library.

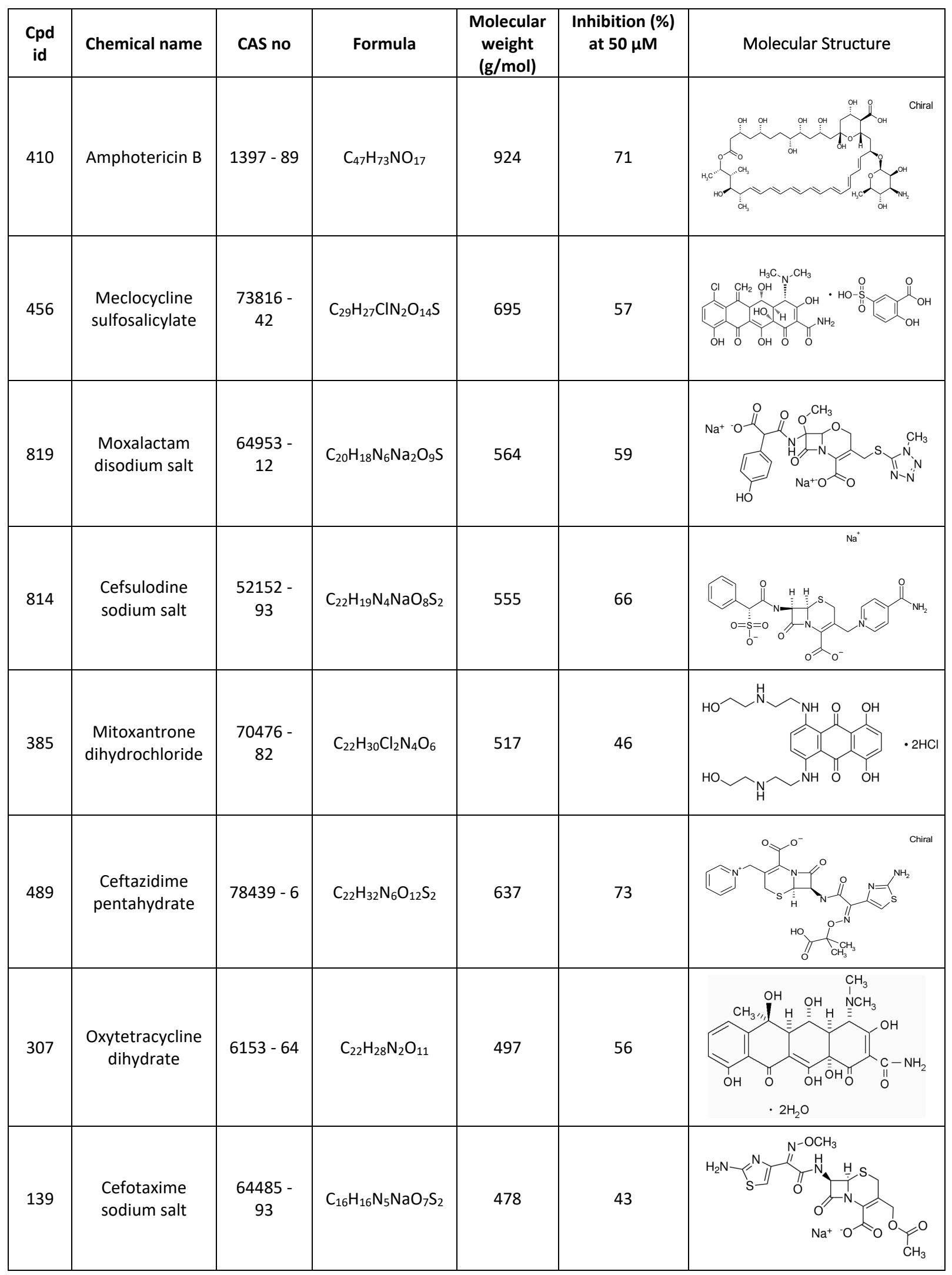




\section{Figure S1}

\section{hNTH1}

Glycosylase activity

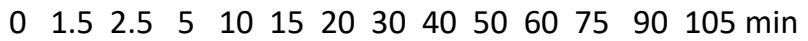

Glycosylase \& AP-lyase activity

$0 \quad 1.52 .55101520304050607590105$ min

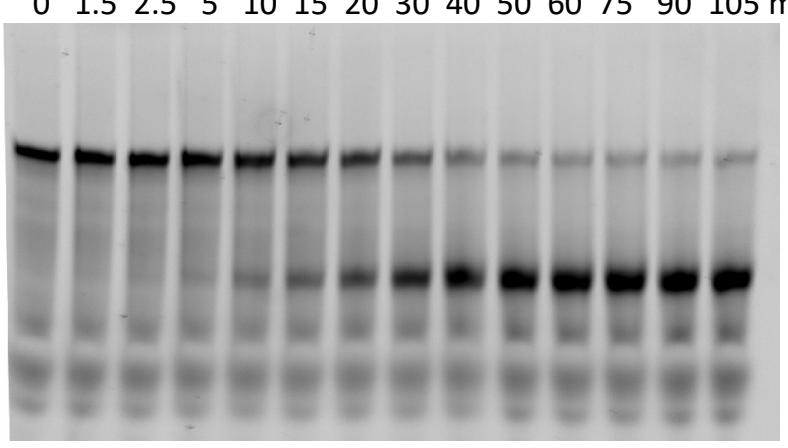

Glycosylase \& AP-lyase activity

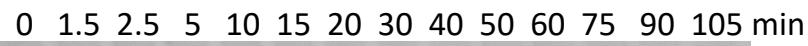

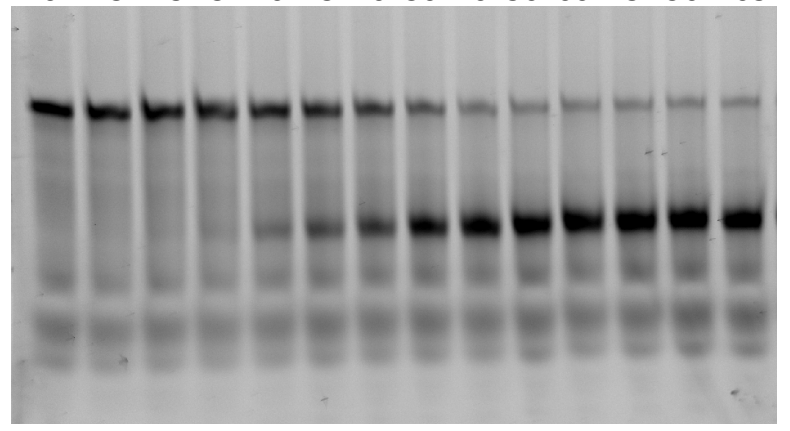

Figure S1. Examples of gels used for measuring the DNA glycosylase and AP-lyase activities of hNTH1 in the absence (upper gels) and presence of an excess of YB1 (lower gels). For the gels on the right, samples were treated with $\mathrm{NaOH}$ before loading them on gel, in order to cause cleavage of all strands bearing abasic sites. This allows to follow the glycosylase activity. In the gels on the left, strand cleavage was performed by the AP-lyase activity of hNTH1 following its removal of the modified base. The DNA strand containing the thymine glycol was labelled with a fluorescein moiety on the 5 ' end and DNA was visualized and quantified on a ChemiDoc MP Imager (Bio-Rad). 


\section{Figure S2}

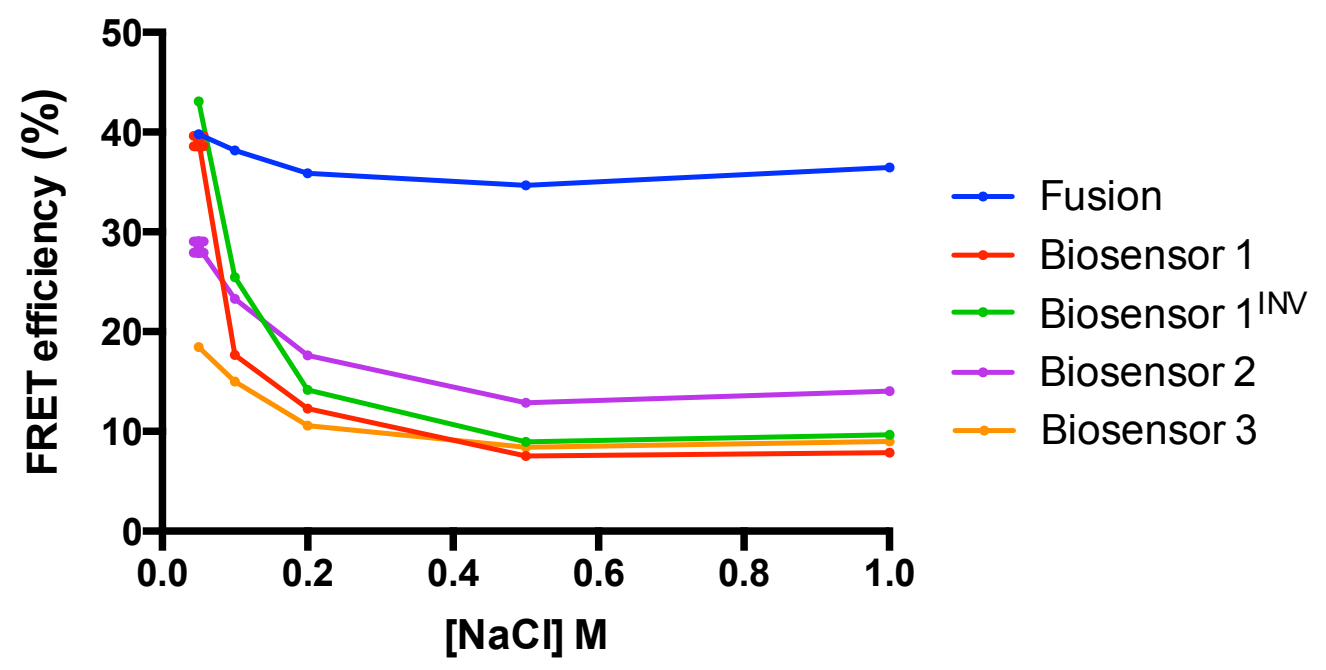

Figure S2. FRET efficiencies of $1 \mu \mathrm{M}$ Fusion and Biosensor constructs at different $\mathrm{NaCl}$ concentrations (50 $\mathrm{mM} \mathrm{NaCl}-1 \mathrm{M} \mathrm{NaCl}$ ). The FRET signal of the Fusion is slightly affected by high salt concentrations (5\% decrease). All Biosensor constructs had a basal FRET signal ranging between 8 and 15\%. Biosensors 1 and $1^{1 \mathrm{NV}}$ showed high FRET efficiencies at $50 \mathrm{mM} \mathrm{NaCl}$, but this efficiency decreased significantly above $0.1 \mathrm{M}$ $\mathrm{NaCl}$, indicating that the interaction between $\mathrm{hNTH} 1$ and $\mathrm{YB} 1 \Delta \mathrm{C}$ is salt-sensitive. 


\section{Figure S3}

A

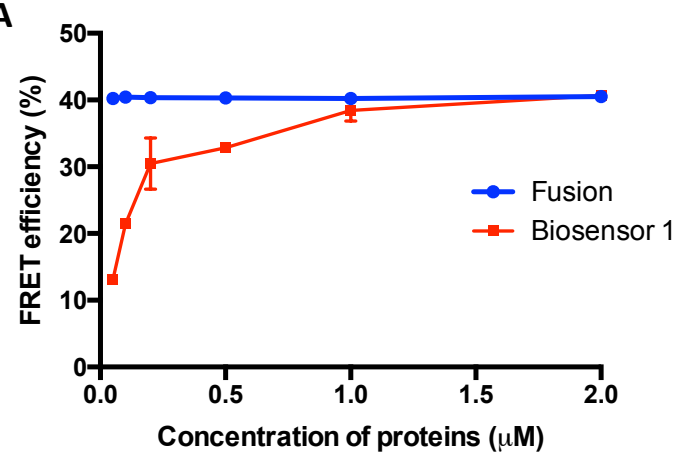

C

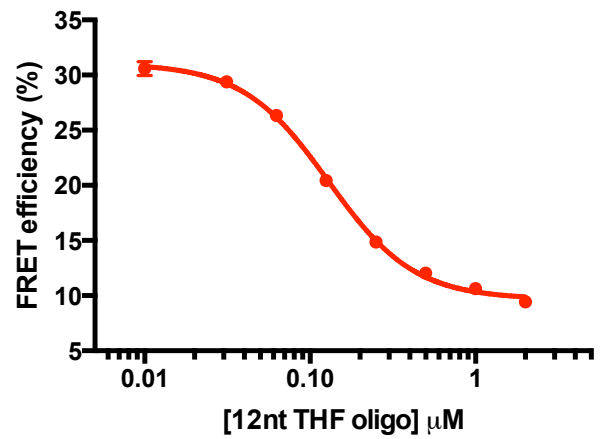

B

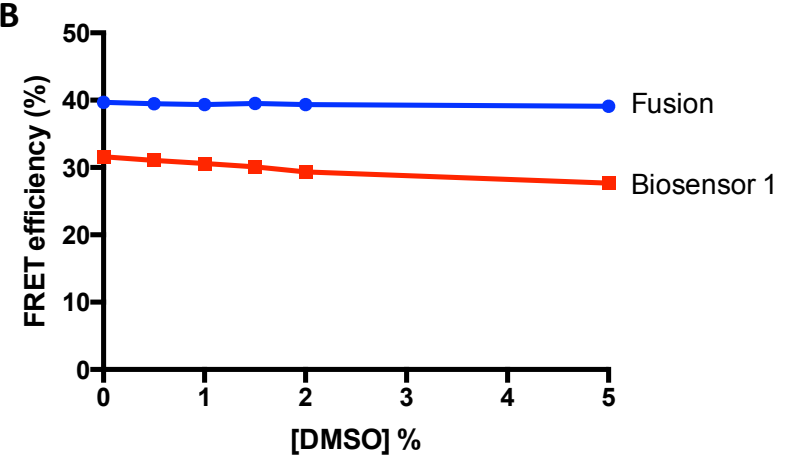

D

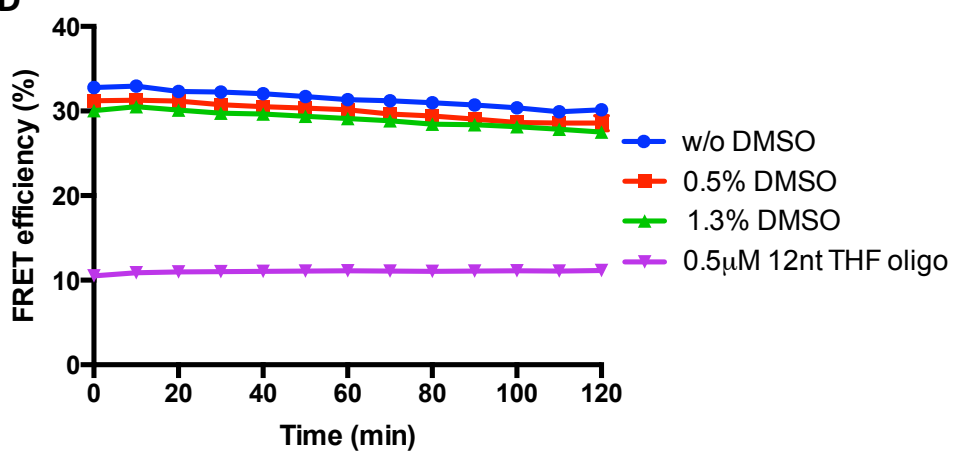

Figure S3. Optimization of Biosensor 1 as a tool for chemical library screening. (A) FRET efficiencies of Fusion and Biosensor 1 constructs at different protein concentrations in buffer containing $50 \mathrm{mM} \mathrm{NaCl}$. The FRET signal of the Fusion was constant regardless of the protein concentration. However, the FRET signal of Biosensor 1 dropped below $0.2 \mu \mathrm{M}$ protein concentration. Subsequent screening experiments were thus performed using $0.2 \mu \mathrm{M}$ Biosensor 1. (B) Stability of the FRET signal of $0.2 \mu \mathrm{M}$ Fusion and Biosensor 1 constructs in the presence of increasing amounts of DMSO $(0.5,1,1.5,2$, and $5 \%)$. A slight decrease in the FRET efficiency of Biosensor 1 was observed at 5\% DMSO. (C) Dose-dependent Inhibition of Biosensor 1 FRET by the hNTH1 DNA substrate: $12 \mathrm{mer}$ THF-oligo. FRET efficiencies of $0.2 \mu \mathrm{M}$ Biosensor 1 in buffer containing $50 \mathrm{mM} \mathrm{NaCl}$ was measured in the presence of $0.01,0.03,0.06,0.13,0.25,0.5,1$, and $2 \mu \mathrm{M}$ THF-oligo. The data points corresponding to the means of triplicate measurements were fitted to a standard sigmoidal inhibition model in GraphPad Prism 6. (D) Stability of the FRET signal of Biosensor 1. The FRET efficiency of Biosensor 1 was monitored over a 2-hour period in buffer containing $50 \mathrm{mM} \mathrm{NaCl}$ (blue), supplemented with either $0.5 \%$ DMSO (red), 1.3\% DMSO (green) or $0.5 \mu \mathrm{M}$ THF-oligo (purple). 


\section{Figure S4}

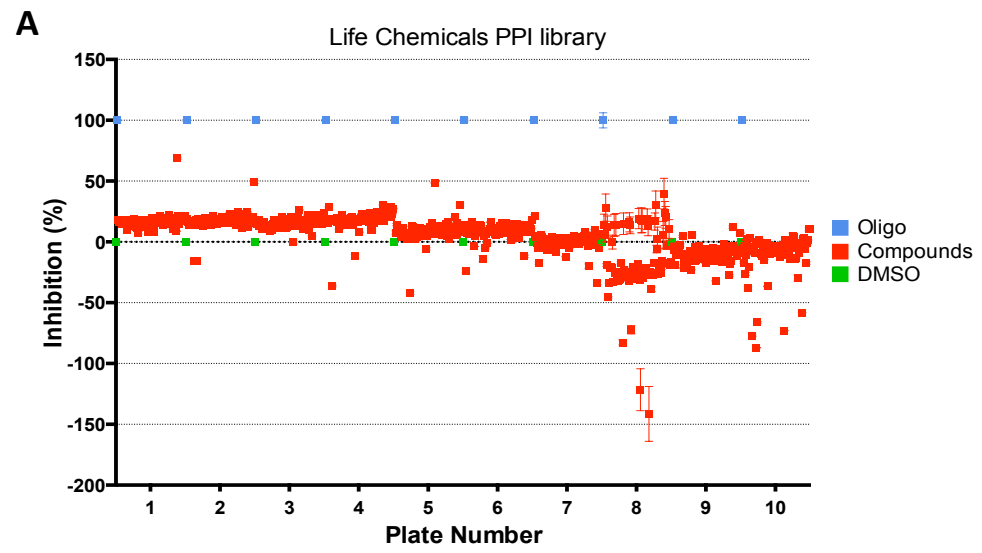

B

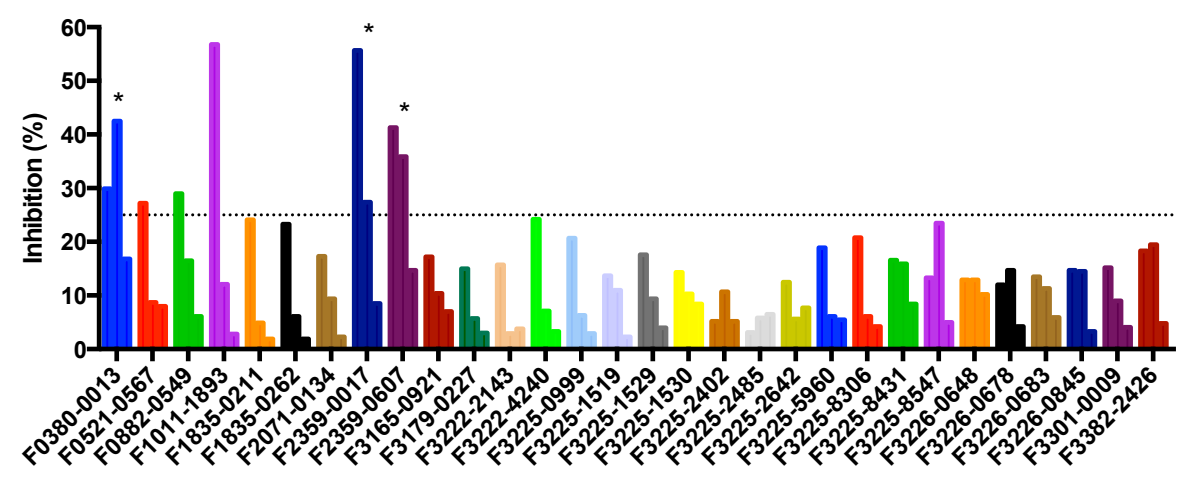

Life Chemicals Compounds

C

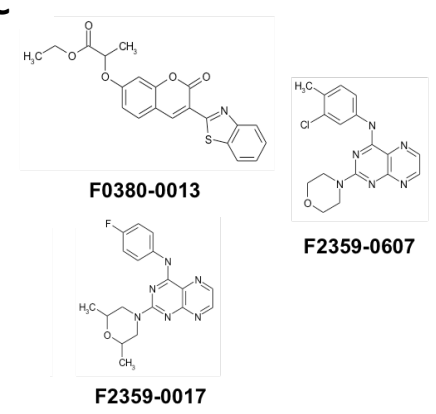

D

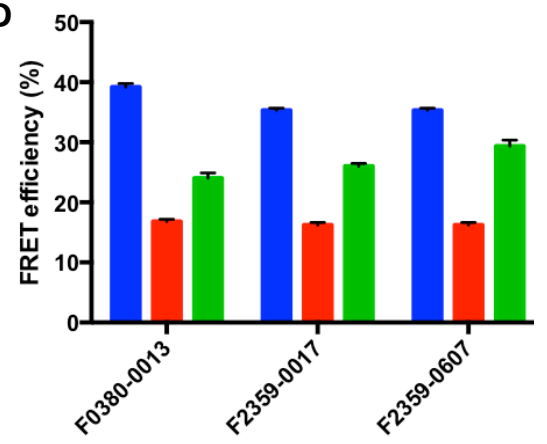

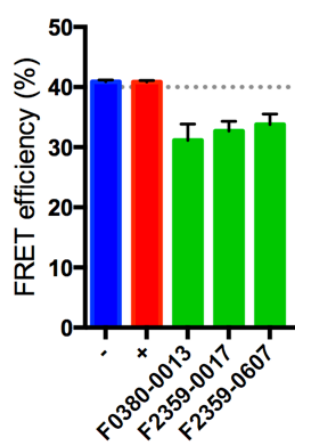

Figure S4. Results of the primary and secondary HTS of the Life Chemicals PPI fragment library and validation of the hits. (A) Overall results obtained from the primary screen. The FRET of Biosensor 1 in DMSO (green dots) was set to 0\% inhibition, while the FRET of Biosensor 1 in the presence of THF-oligo (blue dots) was set to $100 \%$ inhibition. The effects of test compounds at $50 \mu \mathrm{M}$ from ten 384 -well plates are shown as red dots in the graph. (B) Results obtained from the secondary screen of 30 selected compounds selected from the primary screen. The graph presents the extent of inhibition of Biosensor 1 FRET efficiency in the presence of these 30 compounds at 50, 10 and $1 \mu \mathrm{M}$ (left, middle and right histograms, respectively). The dashed line represents $25 \%$ inhibition of Biosensor 1 FRET efficiency. Selected compounds used in further validation tests are marked with an asterisk $\left({ }^{*}\right)$. (C) Chemical structures and references of the three potential hits from the Life Chemicals PPI library. (D) FRET efficiencies of $0.2 \mu \mathrm{M}$ Biosensor 1 (left panel) and Fusion (right panel) in the presence of either 1.3\% DMSO alone (blue), $0.5 \mu \mathrm{M}$ THF-oligo (red) or $50 \mu \mathrm{M}$ compounds identified in the secondary screen (asterisk in (B); green). These initial validation experiments revealed that these were in fact false positives. 
Figure S5

A
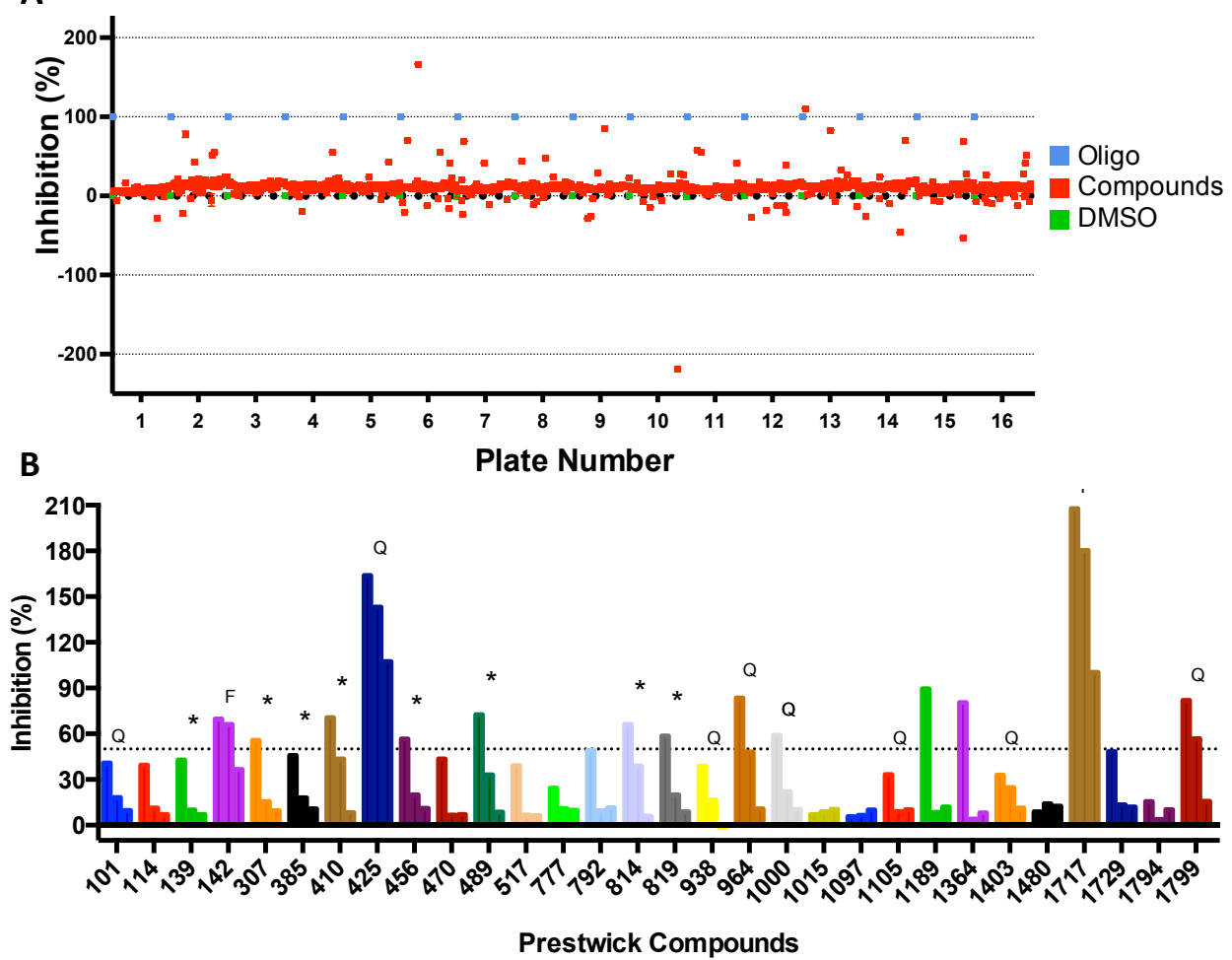

C
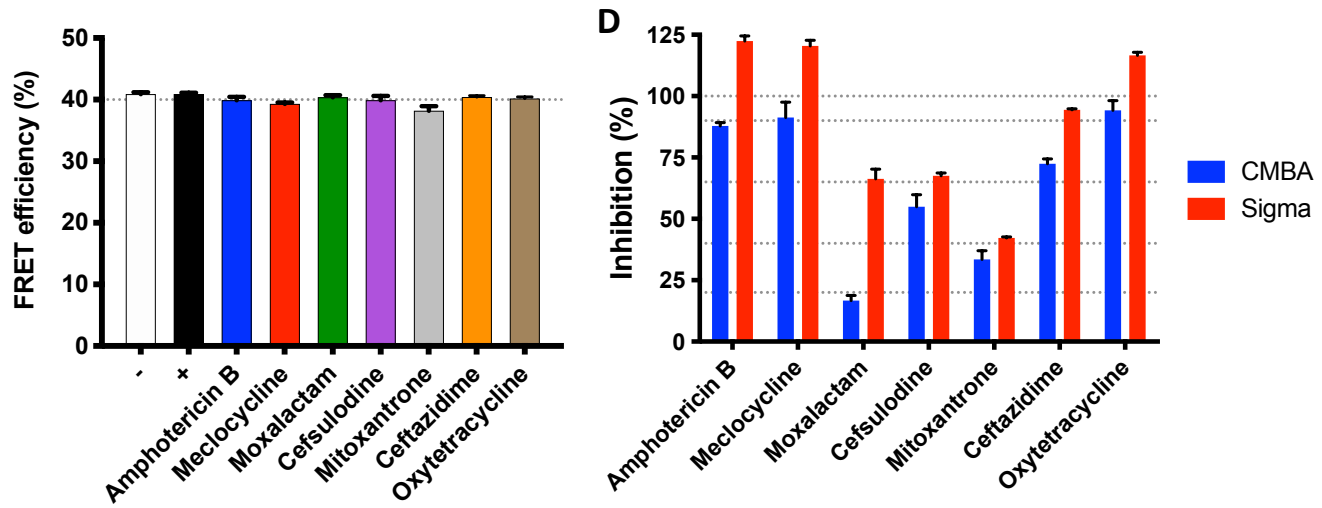

Figure S5. Results of the primary and secondary HTS of the Prestwick Chemical library. (A) Overall results obtained from the primary screen. The FRET of Biosensor 1 in DMSO (green dots) was set to $0 \%$ inhibition, while the FRET of Biosensor 1 in the presence of THF-oligo (blue dots) was set to $100 \%$ inhibition. The effects of test compounds at $50 \mu \mathrm{M}$ from sixteen 384-well plates are shown as red dots in the graph. (B) Results obtained from the secondary screen of 30 selected compounds selected from the primary screen. The graph presents the extent of inhibition of Biosensor 1 FRET efficiency in the presence of these 30 compounds at 50,10 and $1 \mu \mathrm{M}$ (left, middle and right histograms, respectively). The dashed line represents $50 \%$ inhibition. Selected compounds used in further validation tests are marked with an asterisk $(*)$. F indicates the compounds, which showed high intrinsic fluorescence signal alone. $Q$ indicates the compounds that quenched the fluorescence signal. (C) FRET efficiencies of $0.2 \mu \mathrm{M}$ Fusion in buffer containing $50 \mathrm{mM} \mathrm{NaCl}$ in the presence of DMSO (blue), THF-oligo (red) or $50 \mu \mathrm{M}$ test compounds. No significant change was detected on the FRET signal of the Fusion construct in the presence of the seven selected Prestwick compounds. (D) Comparison of the extent of inhibition of Biosensor 1 FRET signal by seven selected Prestwick compounds either provided by the CMBA platform (blue) or prepared inhouse from powders (red). 
Figure S6
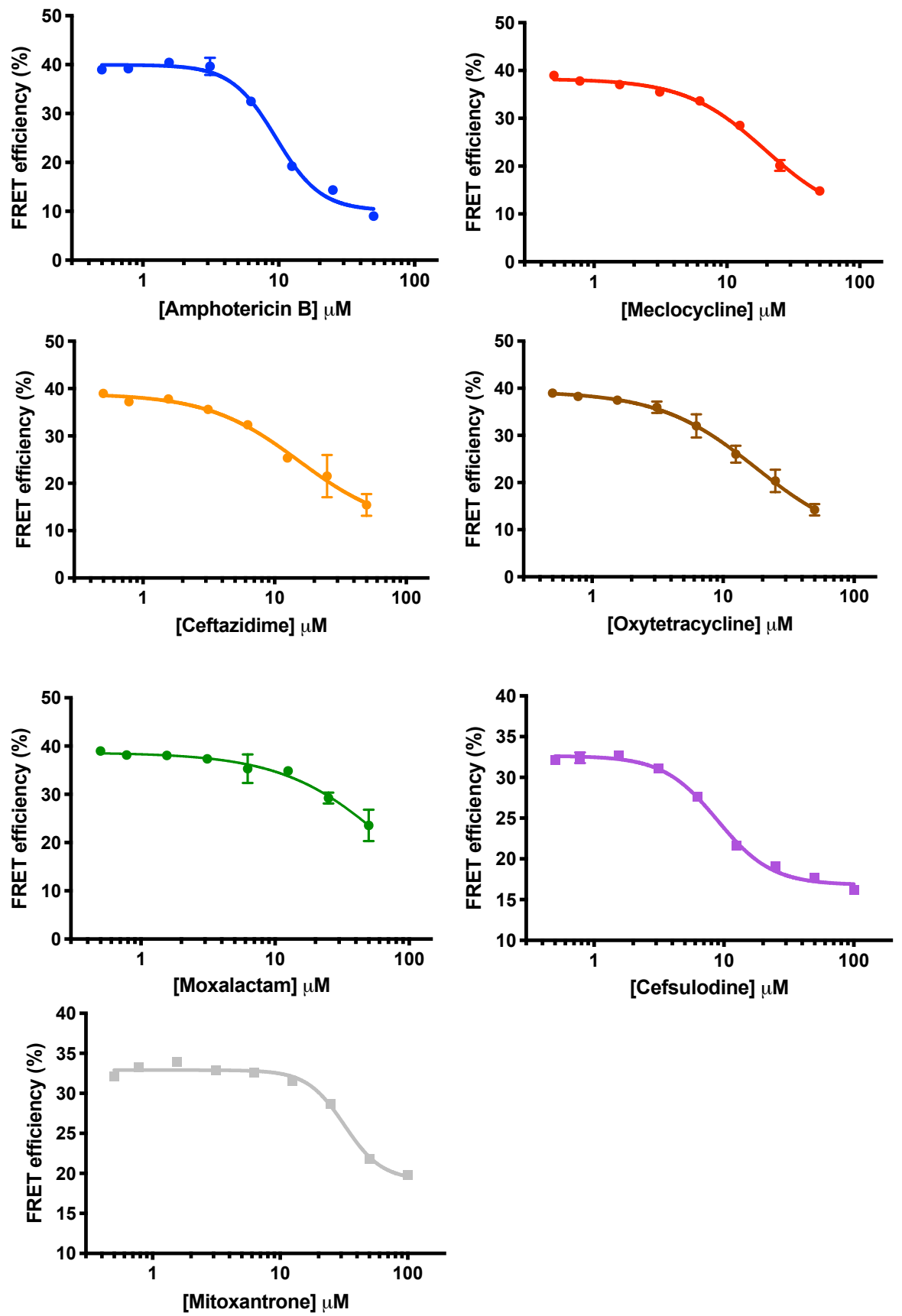

Figure S6. Dose-dependent inhibition of Biosensor 1 FRET efficiency by amphotericin B (blue), meclocycline (red), ceftazidime (orange), oxytetracycline (brown), moxalactam (green), cefsulodine (purple) and mitoxantrone (grey). The data points corresponding to the means of triplicate measurements were fitted to a 4-parameter sigmoidal inhibition model in GraphPad Prism 8 to determine the $\mathrm{IC}_{50}$ values (Table 1 ). 
Figure S7

A

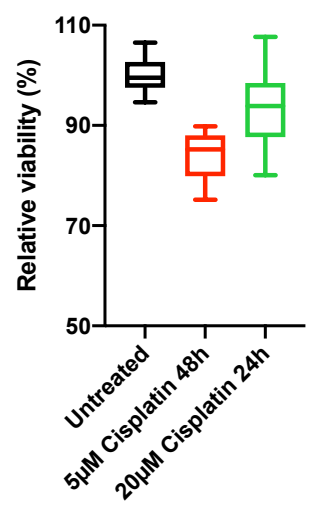

B
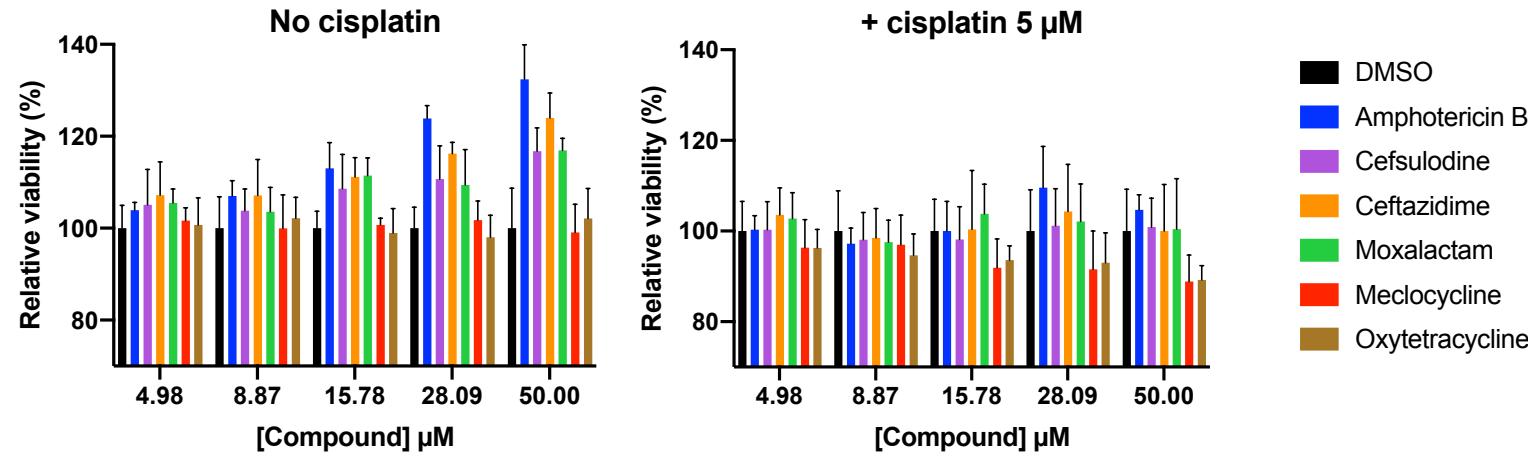

C
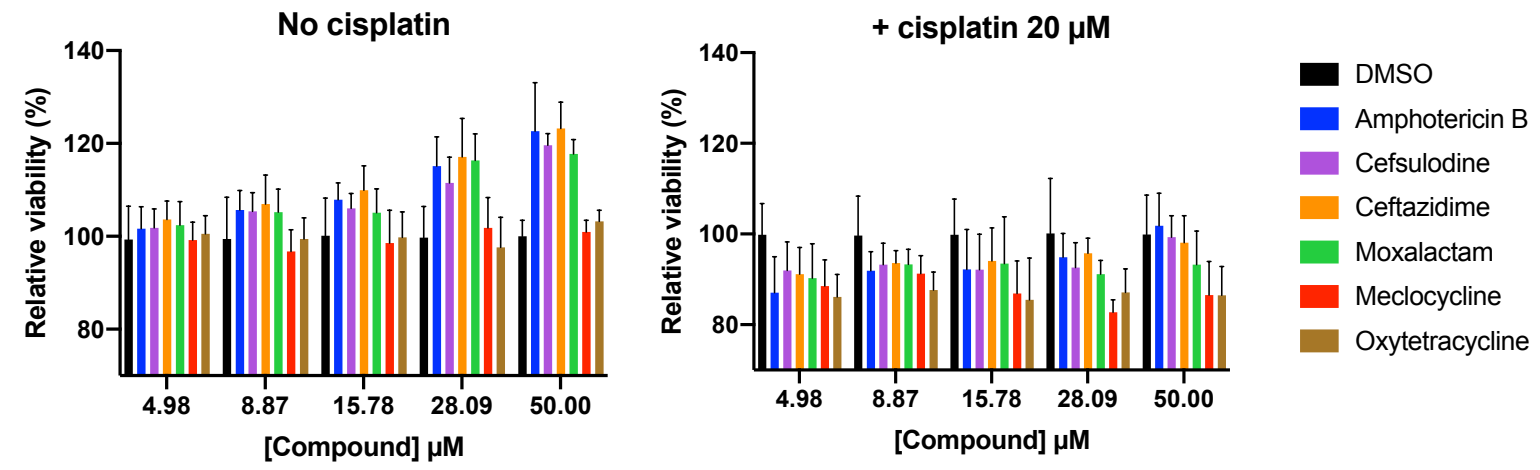

Figure S7: Effects of selected hits on the sensitivity of MCF7 cells to cisplatin. (A) Effects of cisplatin on MCF7 viability. The relative viability of MCF7 cells treated with either 5 or $20 \mu \mathrm{M}$ cisplatin for respectively 48 and $24 \mathrm{~h}$ was compared to that of untreated cells. The box-and-whiskers plot presents the median, $\min$. and max. of 20 replicates. (B) Relative viability of MCF7 cells treated with either 5-50 $\mu \mathrm{M}$ selected compounds or DMSO (black) only (left panel) or with both 5-50 $\mu \mathrm{M}$ selected compounds or DMSO (black) and $5 \mu \mathrm{M}$ cisplatin for $48 \mathrm{~h}$ (right panel). Plotted histograms are the mean of four replicates and error bars represent the standard deviation. (C) Relative viability of MCF7 cells treated with either $5-50 \mu \mathrm{M}$ selected compounds or DMSO (black) only (left panel) or with both 5-50 $\mu \mathrm{M}$ selected compounds or DMSO (black) and $20 \mu \mathrm{M}$ cisplatin for $24 \mathrm{~h}$ (right panel). Plotted histograms are the mean of four replicates and error bars represent the standard deviation. (A)-(C) The viability of DMSO treated cells at each concentration was set to $100 \%$ and was used as a reference for calculating the relative viability of cisplatin and/or compound-treated cells. All graphs were prepared with GraphPad Prism 8. 
Figure S8
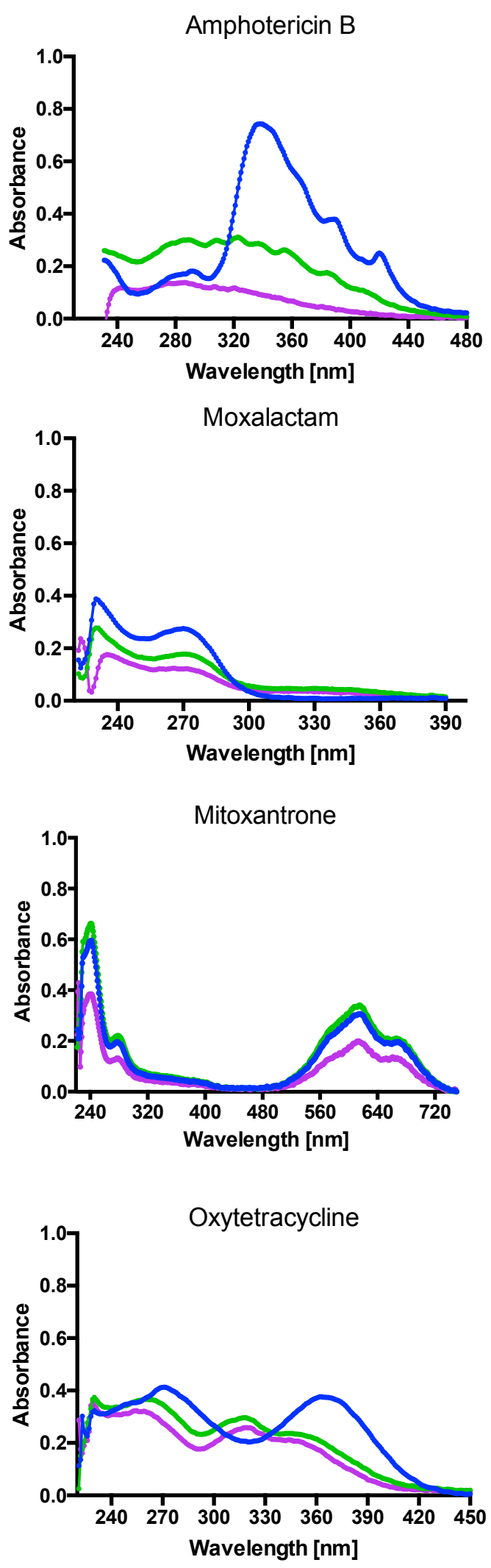
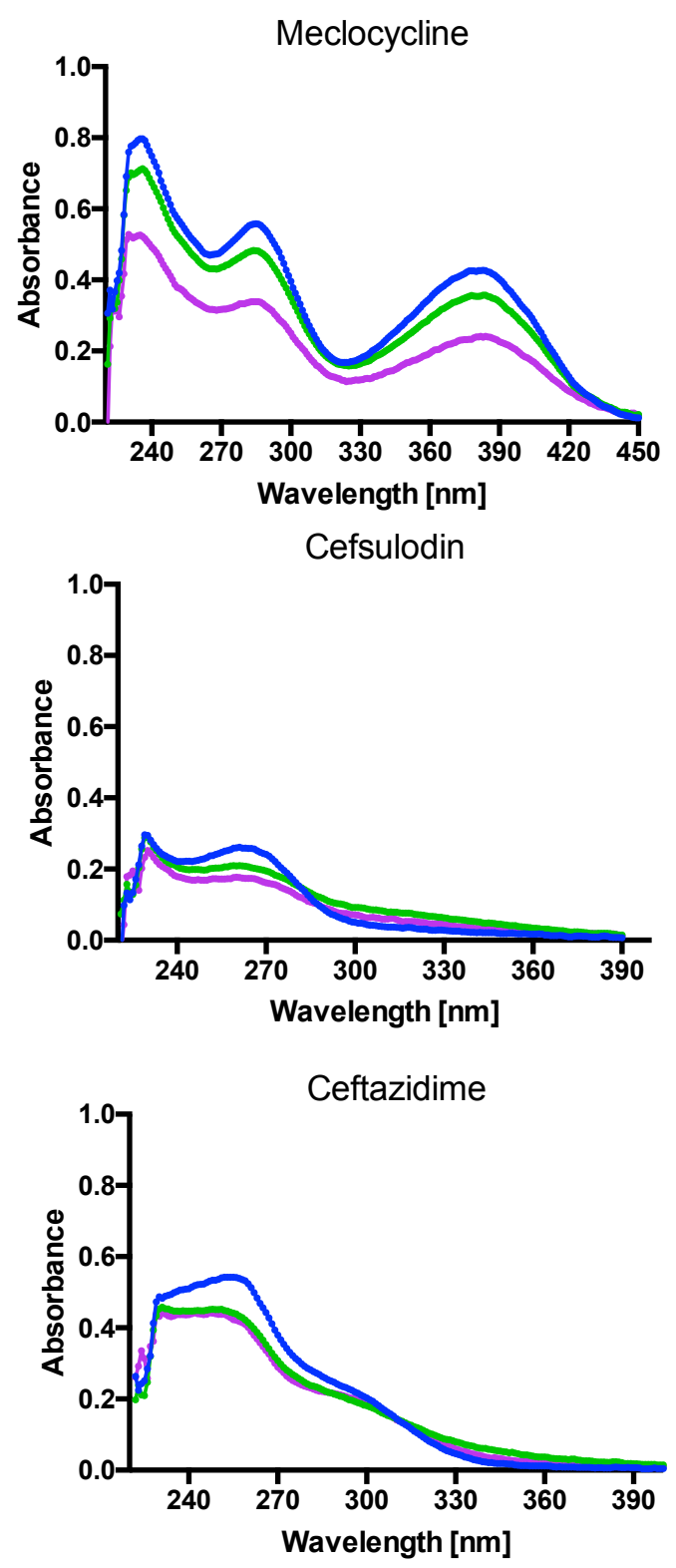

Figure S8. UV/Vis spectral analysis of the seven selected Prestwick compounds. Spectra were performed either on the compound stocks provided by the CMBA platform (purple) or on the compound stocks prepared in house starting from powders (blue and green). The blue spectra were performed on the stock solutions immediately after their preparation, whereas the green spectra were performed on solutions stored at $4^{\circ} \mathrm{C}$ for 7 days. 